\title{
INTERACTIVE AND IMMERSIVE COASTAL HYDRODYNAMIC SIMULATION
}

\author{
Patrick Lynett, University of Southern California, plynett@usc.edu \\ Sasan Tavakkol, University of Southern California, tavakkol@usc.edu
}

\begin{abstract}
INTRODUCTION
In this presentation, we will discuss the development and application of a GPU-based Boussinesq-type wave model. The novelty of this approach is that it is meant to serve the primary purpose of being interactive allowing the user to modify the boundary conditions and model parameters as the model is running, and to see the effect of these changes immediately. To accomplish this, the model is coded in a shader language environment, and our physical variables (e.g. ocean surface elevation, water velocity) are represented in the model as textures, which can be rapidly rendered and visualized via a GPU.
\end{abstract}

This software can help scientists better understand nearshore wave dynamics as it allows them to observe wave interactions in real-time and modify the boundary conditions and model parameters as the model is running to see the effect of these changes immediately. The model is named "Celeris", and is released under the GNU (open-source, open-access) license.

\section{APPROACH}

Celeris uses a hybrid finite volume - finite difference method to solve the extended Boussinesq equations on the GPU. Celeris is validated for three breaking and non-breaking wave benchmarks, as well as for a current benchmark (Tavakkol \& Lynett, 2017). Not only does it run faster than real time for practical engineering simulations, it provides several concurrent visualization options for viewing surface elevation, velocities, and vorticity. We further discuss the recently added features such as ability to adjust tide/surge level without interrupting the model, and the ability to easily modify the wave field through the user interface.

The high performance computing capabilities of the software come from GPU. The simulations are done on GPU using DirectX API. In order to use this API, the computational problem needs to be reformulated in terms of graphics primitives and in accordance with underlying graphical concepts. To solve the equations on the GPU, the simulation grid is assumed as a graphical texture and copied on the GPU. Then each step of the scheme (e.g. reconstruction, flux calculation, etc.) is done by passing a shader over the texture. Shaders are subroutines used to perform computations on each element of texture (texel). This means that thousands of individual GPU cores are simultaneously working on a grid.

\section{APPLICATIONS}

Use of the model for a range of different problems will be presented. The discussion of these examples will focus on the ease-of-setup, the rapid and visual simulation, the interaction with the simulation, and extraction of model output for design purposes. Specifically, we will examine tsunami flooding of an urban environment, hurricane wind wave inundation with variable background sea levels, and integration of the Celeris model with wave forecast products, such that a short-range coastal wave forecast can be provided.

We expect that the Celeris model approach, wherein visualization, interactivity, rapid computation are given equal priority to model accuracy, will open a new application space for simulation within coastal engineering. Collaborative and interactive design of coastal and harbor projects is possible. Instead of performing back-of-the-envelope calculations of, for example, wave height in a marina, this type of Boussinesq simulator can provide site-specific properties immediately. Demonstration of coastal wave and hydrodynamic processes to clients, students, and the general public becomes easy and intuitive.

Finally, we explore the opportunities in immersive visualization of coastal waves through Virtual Reality and Augmented Reality (VR/AR). With VR, it becomes possible be standing on the beach, in a virtual sense of course, during storm conditions or a future high sea level, for example. A designer can use VR to see how a particular coastal engineering project might look from a sitting-in-the-beach-chair or swimming-in-the-water perspective, both during construction and when final.

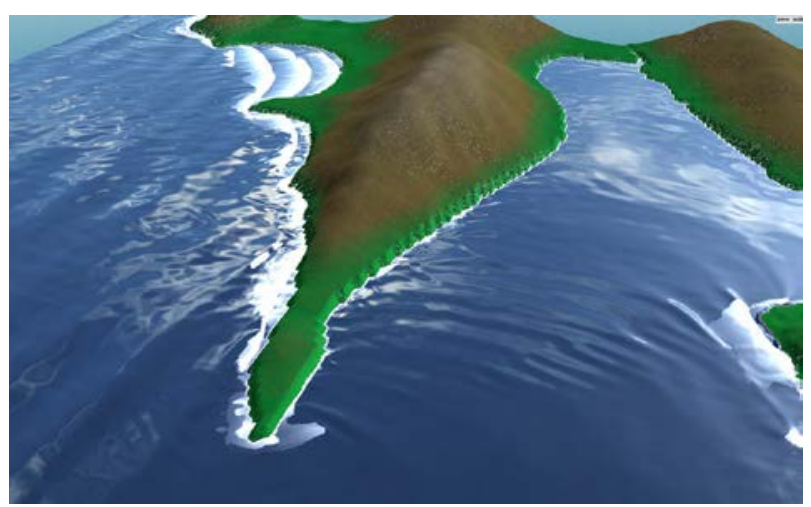

Figure 1 - Example simulation snapshot, showing wave propagation off the west coast of Tortola, British Virgin Islands

\section{REFERENCES}

Tavakkol, S. and Lynett, P. (2017) "Celeris: A GPUaccelerated open source software with a Boussinesqtype wave solver for real-time, interactive simulation and visualization." Computer Physics Communications, doi: 10.1016/j.cpc.2017.03.002. 